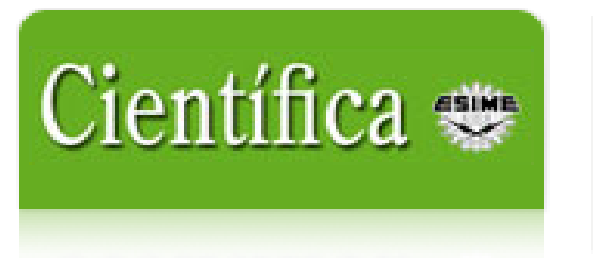

\title{
Científica
}

ISSN: 1665-0654

revista@maya.esimez.ipn.mx

Instituto Politécnico Nacional

México

Escarela Pérez, R.; Aguilar López, R.; Campero Littlewood, E.; Hernández Ávila, J. L.; Avilés Cruz, C.

Finite-Element Calculation of the SSFR of Synchronous Machines

Científica, vol. 10, núm. 2, abril-junio, 2006, pp. 91-102

Instituto Politécnico Nacional

Distrito Federal, México

Available in: http://www.redalyc.org/articulo.oa?id=61410206

How to cite

Complete issue

- More information about this article

Journal's homepage in redalyc.org

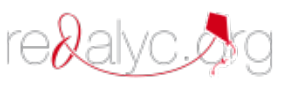

Scientific Information System Network of Scientific Journals from Latin America, the Caribbean, Spain and Portugal Non-profit academic project, developed under the open access initiative 


\section{Finite-Element Calculation of the SSFR of Synchronous Machines}

\section{R. Escarela-Pérez ${ }^{*}$ \\ R.Aguilar-López \\ E. Campero-Littlewood \\ J. L. Hernández-Ávila \\ C. Avilés-Cruz}

Universidad Autónoma Metropolitana, UAM-Azcapotzalco, Av. San Pablo núm. 180, CP 02200, México, DF.

Tel. 5318-9584, 5394-7378 (fax)

*corresponding author.

email: r.escarela@ieee.org

Recibido el 1 de febrero de 2005; aceptado el 8 de noviembre de 2005 .

\section{Abstract}

This paper uses different/state-of-the-art finite-element techniques to effectively obtain the standstill frequency response of synchronous machines. It addresses the continuous interest to avoid actual tests in large machines as they represent high costs and risks of damage. This work uses Lagrange multipliers to create two different finiteelement meshes that are joined at the air gap region, allowing the existence of element nodes in other element edges and facilitates the simulation of the rotor body movement. This is not usually possible in conventional finite-element codes, where the convenient use of dissimilar mesh densities for the stator and rotor components of the machine is not allowed. The finite-element model developed in this work also takes explicit consideration of the external circuitry connected to the machine through a simultaneous and effective solving of the field and circuit equations. The simulations follow the procedure of the corresponding IEEE standard. The numerical results agree well with an older and much less efficient finite-element approach, but that has been fully validated.

Key words: Synchronous Machines; Finite Element Method; Standstill Frequency Response Test.
2. Resumen (El uso de elementos finitos en el cálculo de la respuesta a la frecuencia de máquinas síncronas)

Este artículo usa técnicas modernas de elementos finitos para obtener eficientemente la respuesta a la frecuencia de máquinas síncronas en reposo. Responde al continuo interés que existe por evitar pruebas experimentales en máquinas de alta potencia, debido a que implican altos costos y riesgo de daños. En este trabajo se utilizan multiplicadores de Lagrange para crear mallas diferentes, que se unen en el entrehierro de la máquina, y permiten la existencia de nodos sobre lados de otros elementos y facilitan la simulación del giro libre del rotor. Esto no es realizable con códigos convencionales de elementos finitos, donde la unión de mallas de diferentes densidades para el rotor y el estator no es una opción. El modelo de elementos finitos desarrollado en este trabajo también toma en consideración la circuitería externa conectada a la máquina a través de una solución simultánea de las ecuaciones de los circuitos y del dominio electromagnético. Las simulaciones siguen fielmente los procedimientos de la norma IEEE. Los resultados concuerdan muy bien con una aproximación de elementos finitos obtenida anteriormente, menos eficiente, pero que sirve como marco de comparación, ya que fue completamente validada.

Palabras clave: máquinas síncronas; método del elemento finito; prueba de respuesta a la frecuencia.

\section{Introduction}

Synchronous machines [1] represent the main way to produce substantial amounts of electrical energy. This energy is mostly generated in thermoelectric and hydroelectric plants, where turbines are respectively driven by high pressure steam and falling water in order to convert mechanical into electric energy. The working principles of thermoelectric and hydroelectric generators are the same, but their construction differs somehow due to the markedly distinct speeds found in each type of plant [2]. The synchronous machine has been a subject of research for more than a century, because its electromechanic design can be optimized and due to the growing need of interconnecting more power networks together [3]. The seminal work of Blondel [2] and Park [4] established the basis of the Two-Axis Theory [5], which is 
routinely used nowadays by power system analysts. This way, it is possible to obtain high-order equivalent circuits [6-8] that can be used with circuit simulators to properly design and operate modern power systems. Roughly speaking, a synchronous machine is constituted of copper conductors and ferromagnetic materials arranged in such a way as to enhance magnetic fields interaction, that produce winding voltages and an electromagnetic torque to achieve energy conversion. The nonlinearity of the stator and rotor cores along with the distributed currents induced in the rotor body under transient conditions pose a problem that is difficult to tackle with simple/traditional equivalent circuits since the rotor electromagnetic dynamics cannot be properly modelled [9]. Also, the mechanical system connected to the machine must be appropriately taken into consideration, making the problem a coupled one, where the mechanical and electromagnetic systems must be solved simultaneously. There is an accepted equivalent circuit structure [6] that has been generalized using $n$ damper branches, such that the rotor electromagnetic effects can be reasonably represented. The main problem associated with equivalent circuits is that the identification of the circuit parameters is a task that requires testing of the machine so that the measured data can be used with identification techniques $[8,10,11]$. One of the tests that is predominantly accepted for parameter identification is the Standstill Frequency Response (SSFR) test [12] since it can provide parameters for both axes of the machine. Moreover, the test is performed with low excitation levels as to prevent machine damage. Nevertheless, it is a costly test as it requires that the machine be disconnected of the power network, that is, it must be out of service during the test. In addition, saturation and contact resistances of rotor circuits are not properly taken into consideration and semi-empirical corrections to the circuit parameters must be used to account for this phenomenon [3]. In spite of all these disadvantages is commonly and popularly used to define parameters because it results in equivalent circuits that produce acceptable results when used in power system simulations.

This work shows how state-of-the-art finite-element techniques can be used to model the SSFR test, avoiding in this way the need of actual experimentation avoiding high costs and the possibility of affecting energy supply to consumers. The finite element method [13] is a powerful tool that can be used to directly model the electromagnetic behaviour of electrical machines using partial differential equations that are derived from Maxwell's equations [14]. Synchronous machines have peculiarities that cannot be directly handled with standard finiteelement approaches. For instance, the allocation of windings and rotor motion can pose a special definition of current distribution that is not usually handled by standard codes. Moreover, the conventional finite-element approach requires definition of currents but electrical machines are fed with voltage sources and circuitry, resulting in the need of developing nonconventional methods. This work combines special techniques to obtain an efficient finite-element model. It also shows the use of the theory and the implementation of methods that address the interconnection of external circuitry with the field model, as well as the problem of rotor movement. A «strong» mathematical coupling of the field and circuit equations is performed, whereas rotor motion is taken into consideration using Lagrange multipliers.

\section{The Electromagnetic Equations}

Formal derivation of the partial differential equation that must be solved to obtain the SSFR of a synchronous machine is given in the following paragraphs. The electromagnetic behavior of the synchronous machine is completely described by Maxwell's equations [14]:

$$
\begin{array}{ll}
\nabla \times \mathbf{E}=-\frac{\partial \mathbf{B}}{\partial t} & \nabla \cdot \mathbf{D}=\rho \\
\nabla \times \mathbf{H}=\mathbf{J}+\frac{\partial \mathbf{D}}{\partial t} & \nabla \cdot \mathbf{B}=0
\end{array}
$$

where $\mathbf{E}$ and $\mathbf{H}$ are the electric and magnetic fields. $\mathbf{D}$ and $\mathbf{B}$ are the electric and magnetic flux densities. $\mathbf{J}$ is the free current density and $\rho$ is the free charge density. These equations are complemented with the following constitutive relations to take into account material properties of the medium being analysed:

$$
\begin{aligned}
& \mathbf{D}=\varepsilon \mathbf{E} \\
& \mathbf{B}=\mu \mathbf{H} \\
& \mathbf{J}=\sigma \mathbf{E}
\end{aligned}
$$

where $\varepsilon$ and $\mu$ are the permitivity and permeability, respectively, and $\sigma$ is the conductivity. It is often convenient to find some simplifications in order to solve these two sets of equations when low-frequency electric-power devices are analyzed. One important simplification is established when the displacement current $(\partial \mathbf{D} / \partial t)$ in $(1 \mathrm{c})$ can be neglected under low frequency operation [14]. It is assumed here that the power transfer from stator to rotor in rotating electrical machines takes place instantaneously. Accordingly, $\mathbf{J}$, in equation (1c), represents the current densities impressed from external sources and the internally generated eddy current densities. Another significant simplification is achieved when a two-dimensional approximation of the electromagnetic device is possible. It is a good approximation to consider that the magnetic field distribution of synchronous machines is nearly planar. This 
simplification implies that the vector current densities are axially directed, that is, they only posses one component, resulting into a two-dimensional approach. Hence, the electromagnetic equations can be recast in a more manageable form by using the magnetic vector potential [13, 14]. The magnetic flux density vector can be expressed as the rotational of a potential vector field because of its divergenceless nature (see equation (1d)):

$$
\mathbf{B}=\nabla \times \mathbf{A}
$$

where $\mathbf{A}$ is the magnetic vector potential, which is also axially directed in planar representations. In order to specify completely this vector field, its divergence must also be defined [14]. The divergence of $\mathbf{A}$ in two dimensions is automatically enforced to zero (Coulomb gauge [15]):

$$
\nabla \cdot \mathbf{A}=\frac{\partial A_{z}}{\partial z}=0
$$

since the magnetic vector potential has only one component in the axial direction and it is constant along it. Equations (3) and (1a) can be combined to show that the electric field $\mathbf{E}$ is given by:

$$
\mathbf{E}=-\nabla V-\frac{\partial \mathbf{A}}{\partial t}
$$

where $V$ is the electric scalar potential. It is now clearly seen that equation (5) can be written as a scalar relationship because the electric field has only one component:

$$
E_{z}=-\frac{\partial V}{\partial z}-\frac{\partial A_{z}}{\partial t}
$$

Thus, equations (1c), (2b) and (3) can be combined to give:

$$
\nabla \times\left(\frac{1}{\mu} \nabla \times \mathbf{A}\right)=\mathbf{J}
$$

which can then be rewritten for the two dimensional case as (using (6)):

$$
\left(\nabla \cdot \frac{1}{\mu} \nabla\right) A_{z}=\sigma\left(\frac{\partial V}{\partial z}+\frac{\partial A_{z}}{\partial t}\right)=J_{z}=-\sigma E_{z}
$$

An important property of the electric scalar potential in (6) is obtained by taking the divergence of the axial current density in (8). It can be easily verified that the value of this divergence is zero, since the divergence of the rotational of a vector field is always zero. As a result, the following expression is obtained from the middle term of (8):

$$
\frac{\partial}{\partial z} \sigma \frac{\partial A_{z}}{\partial t}+\frac{\partial}{\partial z} \sigma \frac{\partial V}{\partial z}=0
$$

The first term on the left hand side of (9) is also zero due to (4). Hence the second term of this equation shows that the first partial derivative of $V$ must be a constant and, therefore, $V$ varies linearly in the axial direction. Thus, it can be stated that $\partial V / \partial z$ is simply the potential difference $\left(V_{1}-V_{2}\right)$ measured at the ends of a conducting region divided by the effective conductor length $\left(L_{\text {eff }}\right)$ :

$$
\frac{\partial V}{\partial z}=\frac{V_{1}-V_{2}}{L_{\text {eff }}}=\frac{\Delta V}{L_{\text {eff }}}
$$

It is this potential difference that allows the coupling of the field equations with external electric circuits, as well as, the interconnection between different internal regions of the electromagnetic model.

Equation (8), subject to boundary conditions, represents the solution of the general two-dimensional electromagnetic transient problem. If the electromagnetic system is excited with sinusoidal sources and the material properties ( $\mu$ and $\sigma$ ) have linear characteristics, then (8) can be written in the frequency domain by simple substitution of $\partial / \partial t$ by $j_{\omega}$ :

$$
\left(\nabla \cdot \frac{1}{\mu} \nabla\right) \tilde{A}_{z}=-\tilde{J}_{z s}+j \omega \sigma \tilde{A} z
$$

where

$$
\tilde{J}_{z s}=-\sigma \frac{\partial \tilde{V}}{\partial z}=-\sigma \frac{\tilde{V}_{1}-\tilde{V}_{2}}{L_{e f f}}=-\sigma \frac{\Delta \tilde{V}}{L_{e f f}}
$$

can be thought of as the current density impressed by external sources to the field and stator windings of the machine. The symbol $\sim$ defines a complex quantity. The second term on the right hand side of (11) represents the eddy currents induced in the rotor body and windings of massive conductors [16]. Integration of the right hand side of (11) over coil regions gives the total current externally fed to the machine windings. If there are no eddy currents induced in the machine windings (filamentary coils [17]), it is possible to write (11) as:

$$
\left(\nabla \cdot \frac{1}{\mu} \nabla\right) \tilde{A}_{z}=-\frac{N \tilde{i}}{A_{\text {coil }}}
$$


This equation results from (11) by making the second term on the right hand side of (12) equal to zero and by assuming that the current density $J_{z s}$ over the coil winding is uniformly distributed over its cross section. $A_{\text {coil }}$ is the area occupied by the winding. In any case, the integration of the right hand side of (11) or (13) gives the total winding current $I_{t o t}$.

\section{Finite-Element Approach}

The Finite Element Method is a powerful tool to solve partial differential equations such as (11). In this work, a two-dimensional finite element model with triangular and quadrilateral elements was constructed to solve (11) and obtain the SSFR response of a turbine generator. It is assumed that the permeability pattern of the machine is known and fixed, so that the problem is linear. The magnetic vector potential is approximated in each finite element by:

$$
\tilde{A}_{z}(x, y)=\sum_{i=1}^{h_{e}} N_{i} \tilde{A}_{i}
$$

where $\tilde{A}_{i}$ represents the magnetic vector potential at the $h$ element nodes and $N_{i}$ are shape functions [13]. A hierarchical scheme is used here, where the second order approximation is obtained by adding on new second order terms and degrees of freedom to the first order polynomial expansion [18]. This approximation is similar to adding terms to a Fourier series in order to improve it. The set of complex finite-element linear equations, that represent the original problem (11), can be obtained through the Method of Weighted Residuals [13-15], where the error $(\tilde{\varepsilon})$, produced by assuming a linear variation in each element of the problem domain, is forced to zero in an average sense within the solution domain $(\Omega)$ :

$$
\begin{aligned}
& \int_{\Omega} N_{i}(\tilde{\varepsilon}) d \Omega= \\
& \int_{\Omega} N_{i}\left[\left(\nabla \cdot \frac{1}{\mu} \nabla\right) \tilde{A}_{z}-j \omega \sigma \tilde{A}_{z}+\tilde{J}_{z s}\right]=0
\end{aligned}
$$

where $N_{i}$ is used again here as a weighting function, leading to the well known Galerkin approach [13]. For a first order triangular element, equation (15) can be written at node $i$ as:

$$
\begin{aligned}
& \tilde{\varepsilon}_{i}= \\
& \sum_{j=1}^{3}\left[\frac{1}{\mu}\left[\int_{\Delta e} \nabla N_{i} \nabla N_{i} d \Omega\right] \tilde{A}_{z j}\right. \\
& \left.-j \omega \sigma\left[\int_{\Delta e} N_{i} N_{i} d \Omega\right] A_{z j}\right]-\sigma \frac{\Delta \tilde{V}}{L_{e f f}} \int_{\Delta e} N_{i} d \Omega
\end{aligned}
$$

It has been assumed that the permeability $\mu$ and the current density $J_{z s}$ are uniform over each element. The general system of equations is formed by merging each element contribution to a particular node, leading to a global system of complex equations:

$$
[\tilde{K}]\{\tilde{A}\}+j \omega[C]\{\tilde{A}\}+[Q]\{\Delta \tilde{V}\}=\{0\}
$$

where $[\tilde{K}]$ is a matrix that embodies the first and second terms on the right hand side of (16). $[C]$ and $[Q]$ are matrices that result from the second and third terms on the right hand side of (16). $\{\Delta \tilde{V}\}$ is a vector of potential differences at winding terminals. An additional equation for each winding can be generated by integration of the right hand side of (11):

$$
\int\left|j \omega \sigma \tilde{A}_{z}+\sigma \cdot \frac{\Delta V}{L_{e f f}}\right| d s=I_{t o t}
$$

which can be written in finite-element terms (using a unit weight for the Galerkin formulation [13]), giving a system of equations when all the windings are taken into consideration:

$$
j \omega[Q]^{T}\{A\}+[S]\{\Delta \tilde{V}\}+\left\{I_{t o t}\right\}=\{0\}
$$

where $[S]$ is a matrix that describes the position of the winding terminals. Equations (17) and (19) can be combined, giving the following system of equations:

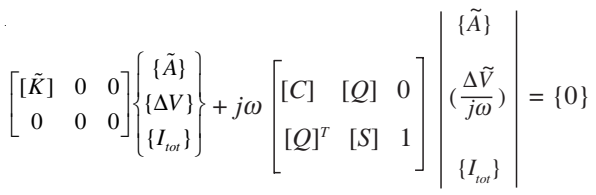

This system of equations must now be completed with the external circuit equations.

\section{Coupling with External Circuitry}

Rotating electrical machines are fed through voltage sources such that winding currents are not known before a finiteelement solution can be obtained. Hence, the third term of (17) is also unknown and therefore the circuit equations of the external devices connected to the machine must be established and solved along with the finite-element system (17) and (19). There are two basic approaches to achieve this goal: 1) a «weak» solution of the two sets of equations and 2) a «strong» coupling of the two sets. The weak approach solves the field equations independently of the circuit ones, 
but the solution of the field equations is used as input data of the circuit equations. Then, the solution of the circuit equations is used as input data of the field system. This process is iterative and performed in the time domain [19]. On the other hand, a strong coupling involves the simultaneous solution of the coupled circuit-field problem. This approach implicates an augmented matrix of the resultant system and proper combination of the field and circuit equations. There are several ways to achieve a strong coupling and several examples can be found in the literature $[20,21]$. Nevertheless, an efficient way of achieving this goal is given here based on [22]. Consider a $R L C$ parallel circuit between nodes 1 and 2, it is not difficult to show through Kirchhoff's nodal equations that:

$$
\left[\begin{array}{ccc}
1 / L & -1 / L & 0 \\
-1 / L & 1 / L & 0 \\
0 & 0 & 1 / C
\end{array}\right]\left(\begin{array}{c}
\int V_{1} d t \\
\int V_{2} d t \\
\int I_{c a p} d t
\end{array}\right)+\left[\begin{array}{ccc}
1 / R & -1 / R & -1 \\
-1 / R & 1 / R & -1 \\
-1 & -1 & 0
\end{array}\right]\left(\begin{array}{c}
V_{1} \\
V_{2} \\
I_{c a p}
\end{array}\right)=\left|\begin{array}{c}
I_{1} \\
I_{2} \\
V_{0}
\end{array}\right|
$$

where $V_{1}$ and $V_{2}$ are the potentials at the terminals of the $R L C$ circuit arrangement. $I_{1}$ and $I_{2}$ are the net currents entering nodes 1 and 2 , respectively. These two currents may be supplied by an external source to the $R L C$ arrangement. $I_{c a p}$ is the capacitor current and $V_{0}$ is the capacitor initial voltage. Now suppose that these elements are to be connected between terminals 1 and 2 of a finite-element winding. Then, the system of equations (20) is complemented in the following form:

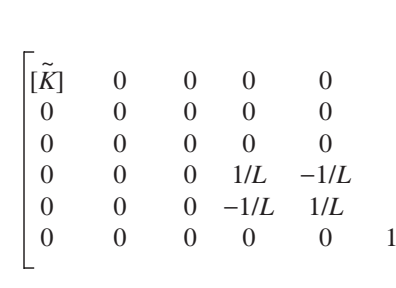$$
\left.\begin{array}{c}
0 \\
0 \\
0 \\
0 \\
0 \\
I^{\prime}
\end{array}\right]\left[\begin{array}{c}
\{\tilde{A}\} \\
\Delta \tilde{V} / j \omega \\
I_{t o t} \\
V_{1} / j \omega \\
V_{2} / j \omega \\
I_{c a p} / j \omega
\end{array}\right]+
$$$$
j \omega\left[\begin{array}{cccccc}
{[C]} & {[Q]} & 0 & 0 & 0 & 0 \\
{[Q]^{T}} & {[S]} & 1 & 0 & 0 & 0 \\
0 & 1 & 0 & -1 & 0 & 0 \\
0 & 0 & -1 & 1 / R & -1 / R & -1 \\
0 & 0 & 0 & -1 / R & 1 / R & 1 \\
0 & 0 & 0 & -1 & 1 & 0
\end{array}\right]\left[\begin{array}{c}
\{\tilde{A}\} \\
\Delta \tilde{V} / j \omega \\
I_{\text {tot }} \\
V_{1} / j \omega \\
V_{2} / j \omega \\
I_{\text {cap }} / j \omega
\end{array}\right]=\left[\begin{array}{c}
0 \\
0 \\
0 \\
0 \\
0 \\
V_{0}
\end{array}\right]
$$

Here, it has been assumed that only one winding exists inside the electromagnetic device. A solution of this problem is obtained when the initial voltage of the capacitor is specified. Additional circuits are added in the same fashion.

\section{Coupling Meshes of Different Densities}

Motion is intrinsic to most electromagnetic devices. Motors and generators have a stationary part, called stator, and a rotating structure called rotor. The stator and rotor are separated by an air gap. The rotor speed can be constant or variable. Hence, it is necessary to take into consideration the rotor motion within the finite-element model. A static mesh would be destroyed by the movement of the rotor and methods to avoid this must be devised. One possible method would be to have two finite element meshes, one for the stator and another for the rotor, joined by a slip boundary at the air gap [23]. This boundary is divided into equal intervals such that rotation can be simulated if nodes in the rotor side always coincide peripherally with nodes on the stator side. This means that rotor nodes belonging to the slip boundary are not allowed to fall between sides of elements in the stator side. Thus, with this technique the topology of the stator and rotor meshes is unchanged, but the time step must be adjusted if speed is allowed to vary arbitrarily. Another approach can be formulated using finite elements in all the machine materials but the air gap, where an analytical solution to Laplace's equation can be found and combined with the finite element part that models the rest of the machine [24]. This permits a smooth motion of the rotor, but this method results in a very non-conventional finite-element approach due to the coupling of the analytical solution with the finite-element discretization.

A method analogous with the «slip boundary» approach is devised by defining a band of elements in the machine air gap. The element band topology is changed as the rotor changes its position. This method is known as the «moving band» technique [25]. Thus, the rotor and stator meshes remain unaltered but the elements in the air gap are distorted until a re-meshing of the band becomes unavoidable.

The slip boundary method can be elegantly modified to enable boundary nodes on the rotor mesh to fall between sides of elements on the stator mesh, this happening at the sliding interface. The magnetic vector potential of both meshes is then coupled using Lagrange multipliers [26]. This technique offers the advantage of avoiding re-meshing. Moreover, the stator mesh can have a very different element density to the density of the rotor mesh. This approach is used here and can be set in the following way. The solution of the field equation (11) can be obtained through the minimization of the co-energy of the electromagnetic domain, that is, the function that minimises the co-energy functional has the additional property of satisfying (11) [15]. This way of solving partial differential equations is mathematically justified with the calculus of variations [15]. The co-energy functional of a domain can be divided into zones and added together to obtain the total co-energy of the system. For 
the rotating machine two sub-domains have been defined: the rotor and stator regions. Both regions are bounded by a band of air and joined together at a circular interface $\Gamma$ within the air gap (see figure 1). Hence the total co-energy can be written as:

$$
W^{\prime}(\tilde{A})=W_{r}^{\prime}(\tilde{A})+W_{s}^{\prime}(\tilde{A})
$$

where $W_{r}^{\prime}(A)$ is the co-energy of the rotor domain whereas $W_{s}^{\prime}(A)$ is the co-energy of the stator region. Minimization of this co-energy functional leads to the sough solution. However, this simple approach is not complete since the system is constrained to make the magnetic vector potential continuous at the circular interface:

$$
\tilde{A_{s}}-\tilde{A_{r}}=0 \quad \text { on } \Gamma
$$

where $\tilde{A}_{s}$ and $\tilde{A}_{r}$ are the potentials that must be matched at the interface. A minimization constrained problem can be tackled and solved using Lagrange multipliers [27], which lead to an equivalent minimization problem that can be stated as:

$$
F(\tilde{A}, \lambda)=W_{r}{ }^{\prime}(\tilde{A})+W_{s}{ }^{\prime}(\tilde{A})+\int \lambda\left(\tilde{A_{s}}-\tilde{A_{r}}\right) d \Gamma
$$

The two first terms on the right hand side of (25) are tackled with standard finite-element techniques that result in a matrix system represented by (17). If there are external devices, they are incorporated using the approach presented in the previous section. The line integral of (25) is performed over the whole circular interface and $\lambda$ is a Lagrange multiplier function that can be put in finite element terms by assuming that

$$
\lambda=\sum_{i} N_{L i} \lambda_{i}
$$

Thus, the Lagrange function has also been discretized using shape functions $N_{L i}$. This shape functions can be defined using the already available discretization of the rotor or stator domain. In other words, the element edges and nodes of the chosen domain that face the circular interface are used to define the Lagrange shape functions. In any case, the integral is finally written in finite element terms as:

$$
\int \lambda\left(\tilde{A}_{s}-\tilde{A}_{r}\right) d \Gamma=\sum_{i} N_{I, i} \lambda_{i}\left(\sum_{s} N_{s} A_{s}-\sum_{r} N_{r} A_{r}\right)
$$

which must be differentiated with respect to all the potentials in both regions, and also with respect to the Lagrange multipliers $\lambda_{i}$. Thus, matrix entries that must be added to (17) are generated.

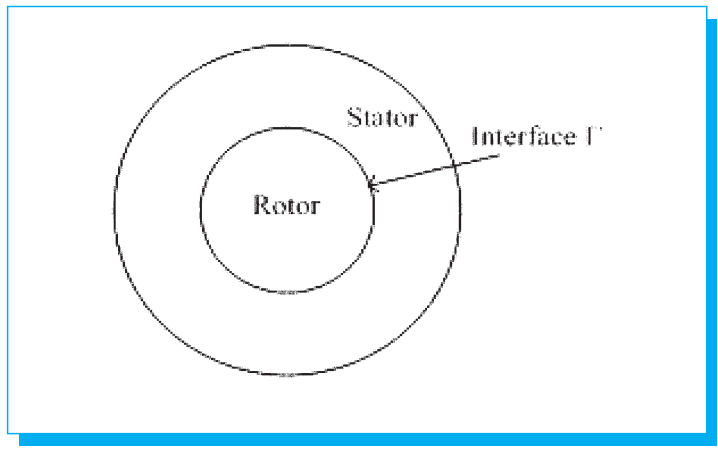

Fig. 1. Connection of stator and rotor domains at $\Gamma$.

\section{Synchronous Machine Finite-Element Model and Transfer Functions}

The design data of a $150 \mathrm{MVA}, 13.8 \mathrm{kV}, 50 \mathrm{~Hz}$ turbine generator was available, so that it was possible to construct a timeharmonic finite-element model and obtain its transfer functions by post-processing of the solution. It is important to mention that this machine was part of Great Britain's power network. The machine geometry is shown in figure 2 , where the circular Lagrange interface can be readily distinguished. This way, it is possible to put the rotor in any position. Particularly in this work the rotor has to be positioned such that the stator field is aligned with the $d$ and $q$ axes. The distribution of the stator phase conductors can be appreciated in figure 2 as well. Figure 3 depicts the finite-element mesh. Two meshes (one for the rotor and one for the stator) were constructed with different densities and coupled at the air gap using Lagrange multipliers as explained in the previous section. Figure 4 shows a close up of the air gap, where the coupling interface and the difference in mesh densities can be seen. A Dirichlet boundary condition [13] was enforced at the outer diameter of the stator. Since the magnetic field is periodic, it is only required to model one half of the full geometry. This periodic condition states that the magnetic vector potential along any radial line must be exactly the negative at an angular displacement of $\pi$, that is:

$$
\tilde{A}(r, \theta)=-\tilde{A}(r, \theta+\pi)
$$

Hence, the radial lines, that serve as boundaries of the rotor and stator geometry (figure 2), are enforced to the periodic boundary condition. 


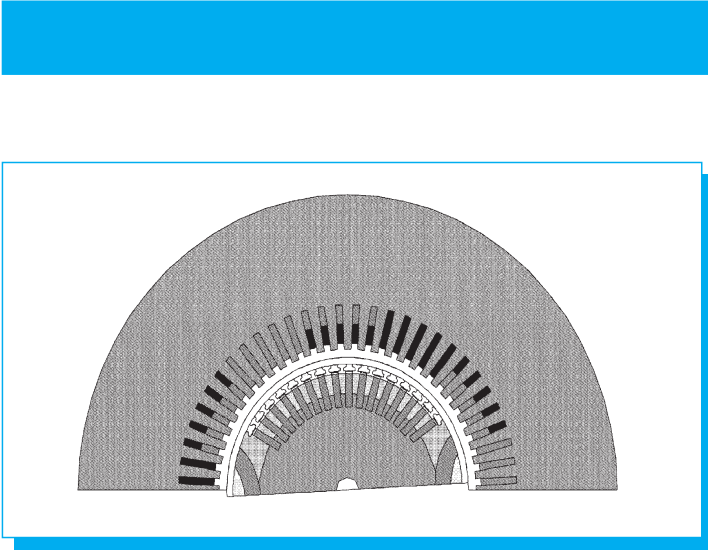

Fig. 2. Machine geometry. One pole pitch.

The SSFR test [12] requires current excitation at the stator terminals, so no coupling with external circuits is required. The rotor/field winding must be open or short circuited, depending on the transfer function that is to be determined. In the first case there is no need to consider coupling with external circuitry, whereas the short circuit condition demands the series connection of the winding resistance and overhang leakage inductance with the field winding modelled within the finite element model. This is achieved with the methodology given in section 5. A constant permeability value, typical of SSFR testing [28], was used to represent the rotor body. The unsaturated permeability value of the stator normal BH curve was used for the stator core [28]. This allows studying the machine under linear conditions. The armature resistance $R_{a}$ and field winding resistance $R_{f}$ are assumed to be known parameters from design data.

The most recent IEEE standard (115-1995 [12]) for performing the SSFR test specifies the connections shown in figures 5 and 6 to obtain the three transfer functions that fully

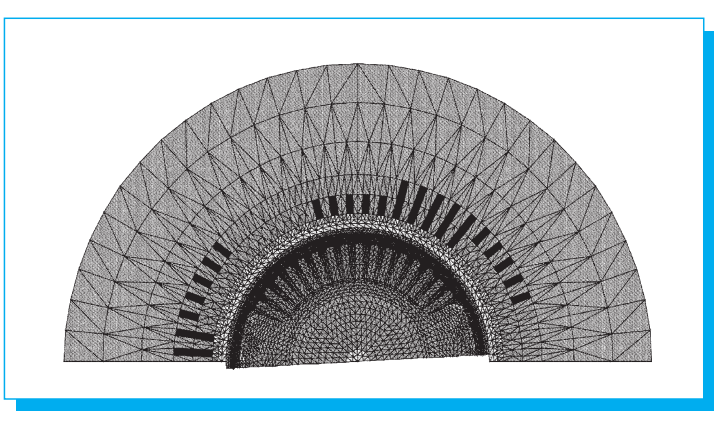

Fig. 3. Finite element mesh.

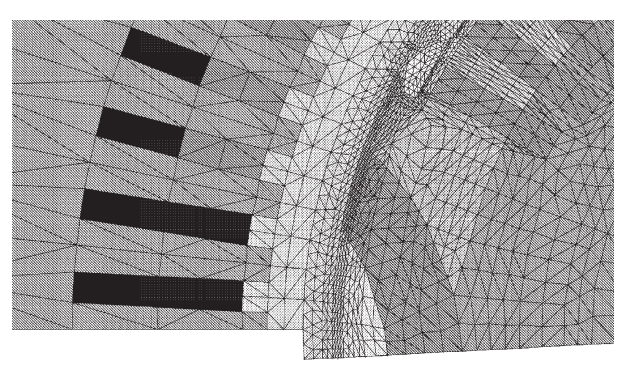

Fig. 4. Mesh zoom of the air gap region showing the different mesh densities of the rotor and stator domains.

characterize the two port network nature of the $d$ axis and to obtain the transfer function of the $q$ axis. Figure 6 applies to the $q$ axis when the field winding is rotated 90 degrees. The transfer functions to be determined are:

$$
\begin{aligned}
& Z_{d}(s)=\left.\frac{\Delta e_{d}(s)}{\Delta i_{d}(s)}\right|_{\Delta e_{j=0}} \\
& s G(s)=\left.\frac{\Delta i_{f d}(s)}{\Delta i_{d}(s)}\right|_{\Delta e_{j d=0}} \\
& Z_{a f o}(s)=\left.\frac{\Delta e_{f d}(s)}{\Delta i_{d}(s)}\right|_{\Delta i_{f d=0}}
\end{aligned}
$$

where $\Delta e_{d}$ and $\Delta i_{d}$ are the $d$-axis voltage and current at the machine terminals of the two-axis transformed machine, whereas. $i_{f d}$ is the current of the field winding. The $\Delta$ symbol emphasises the fact that the tests is performed with small excitations of the stator currents [12], which in turn leads to small values of the induced voltages and currents of the two port network. $s$ is the Laplace's operator and takes the form $j_{\omega}$ in the frequency domain. $Z_{d}$ is the $d$-axis operational impedance. $s G(s)$ is the $d$-axis stator current to field current transfer function. $Z_{a f 0}$ is the armature to field transfer impedance. The $q$-axis quadrature operational impedance is calculated with (29a) when the subscript $d$ is substituted by $q$. Since the synchronous machine is modelled in the $a b c$ reference frame, the transfer functions cannot be evaluated directly and an intermediate impedance and a transfer functions are first calculated. The actual armature impedance that can be calculated from the test measurements is:

$$
Z_{\text {armd }}(s)=\frac{\Delta V_{\text {armd }}(s)}{\Delta i_{\text {armd }}(s)}
$$




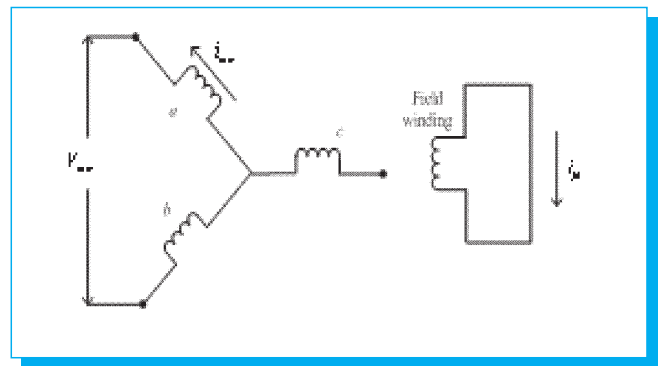

Fig. 5. Connections to obtain $d$-axis transfer functions: field winding short circuited.

such that the $d$-axis operational impedance is given by:

$$
Z_{d}(s)=\frac{1}{2} Z_{\text {armd }}(s)
$$

This armature impedance is divided by 2 because two stator windings are used to perform the test. An alternative transfer function that deserves consideration is the $d$-axis operational reactance, which is given by the following expression:

$$
X_{d}(s)=\left(\frac{Z_{d}(s)-R_{a}}{s}\right) \omega_{0}
$$

where $\omega_{0}$ is nominal angular speed of the machine. This reactance function can be more conveniently used in the identification of equivalent circuits instead of the impedance transfer function [29]. The $d$-axis stator current to field current transfer function is calculated with:

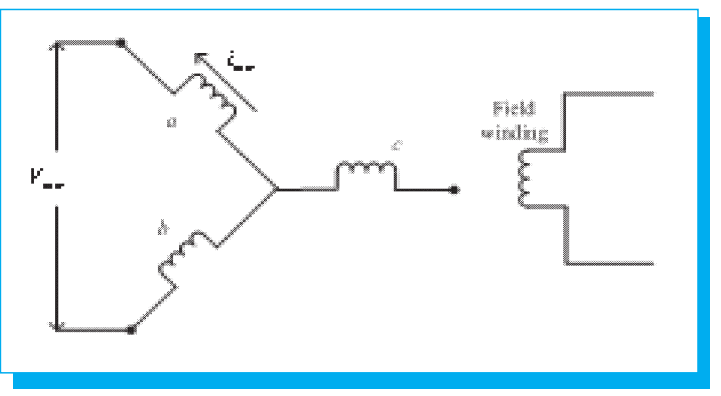

Fig. 6. Connections to obtain $d$-axis transfer functions: field winding open.

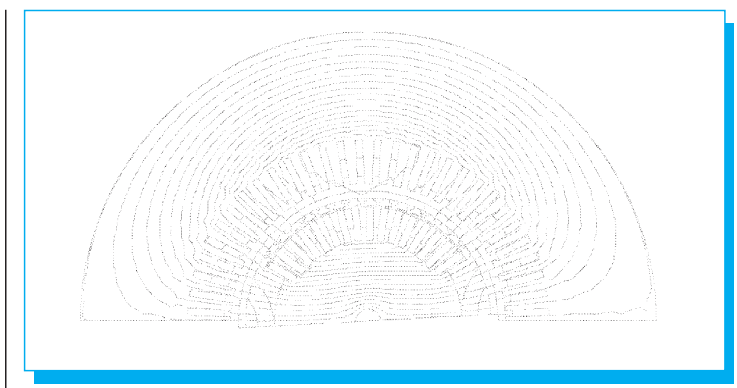

Fig. 7. Magnetic flux density distribution $\left(\omega t=0^{\circ}\right)$ at $0.001 \mathrm{~Hz}$.

$$
s G(s)=\frac{\Delta i_{f d}(s)}{\Delta i_{d}(s)}=\frac{\Delta i_{f d}(s)}{\Delta i_{a r m}(s) / \cos 30^{\circ}}
$$

The $\cos 30^{\circ}$ takes account of Park's transformation from the $a b c$ to the $d q$ frame of reference. Finally, the armature to field transfer impedance is obtained from:

$$
Z_{a f o}(s)=\frac{\Delta e_{f d}(s)}{\Delta i_{d}(s)}=\frac{\Delta e_{f d}(s)}{\Delta i_{a r m}(s) / \cos 30^{\circ}}
$$

A convenient operational inductance can be calculated from (34):

$$
X_{a f 0}=\left(\frac{Z_{a f o}(s)}{s}\right) \omega_{0}
$$

This reactance is called armature to field transfer reactance which also facilitates the identification process of equivalent circuit parameters [29].

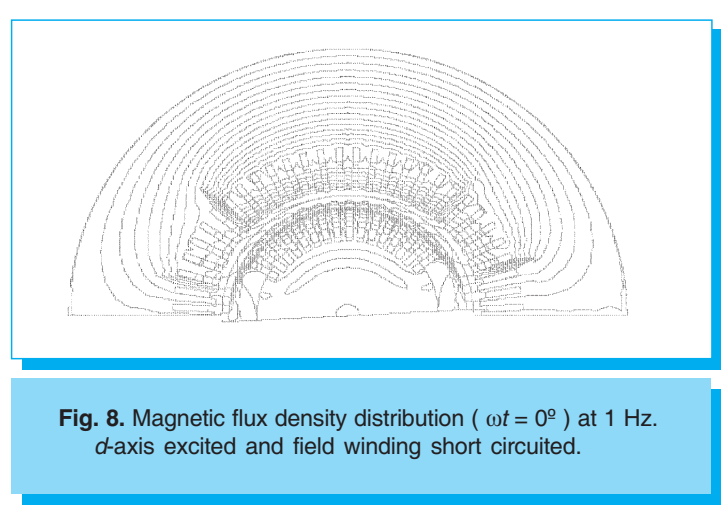




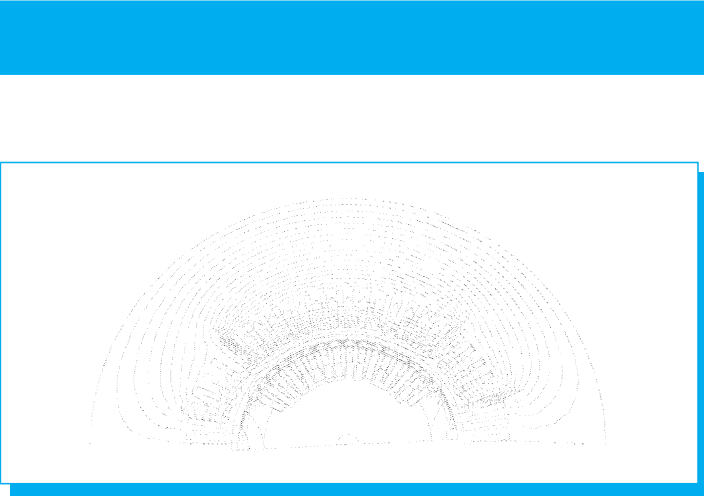

Fig. 9. Magnetic flux density distribution $\left(\omega t=0^{\circ}\right)$ at $10 \mathrm{~Hz}$ $d$-axis excited and field winding short circuited.

\section{Results and Validation}

The finite-element model gives values of the magnetic vector potential at element nodes, whereas currents and voltages are outputs at nodes of the external circuitry, which have been interconnected with the field equations. These values of currents and voltages are those that would be measured in a real test and they are inputted to the transfer function expressions of the previous section to evaluate them. The SSFR test is simulated using a frequency range that goes from 0.001 to $100 \mathrm{~Hz}$, thus complying with the IEEE standard. Figures 7 to 14 show the magnetic flux density distribution obtained from the finite-element solution at different frequencies for the $d$ and $q$ axes. These figures show how the magnetic field is expelled from the rotor body as the frequency is increased. This phenomenon is explained by the fact that the field and eddy currents are induced in such way that the stator magnetic field cannot get into the rotor body. This also conforms to electromagnetic theory results that state that the penetration of an alternating magnetic

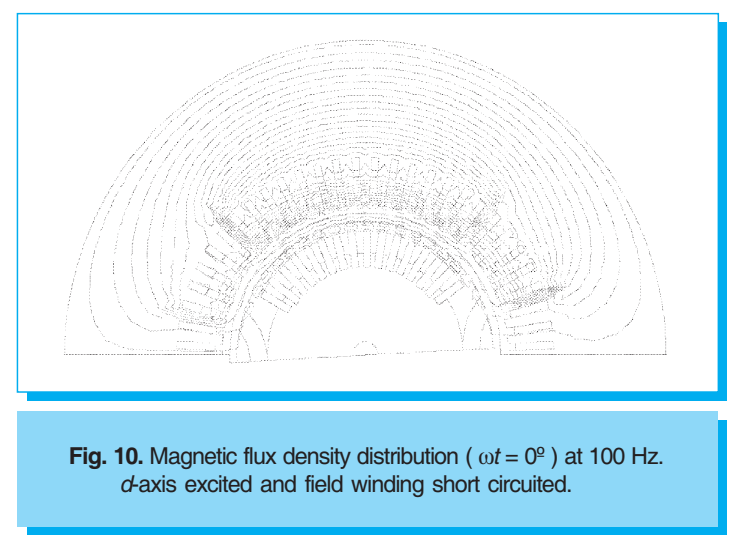




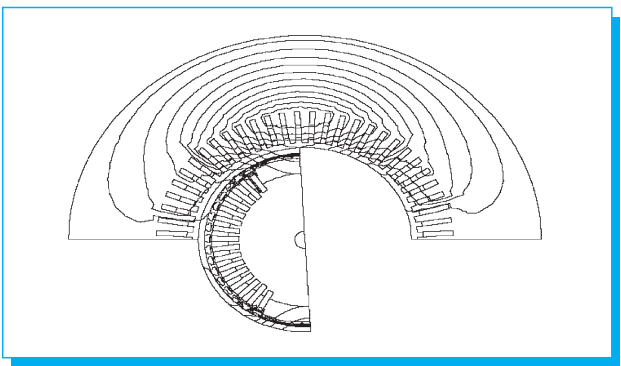

Fig. 13. Magnetic flux density distribution ( $\omega t=0^{\circ}$ ) at $10 \mathrm{~Hz}$. q-axis excited

«old method» refer to these transfer functions. Curves labelled as «new approach» refer to the techniques described in this work. It is important to emphasize that the comparison is shown here since the work of reference [30] was validated and therefore the new results can in turn be validated as well. Nevertheless, it is interesting to notice that the finite-element model of [30] does not model the machine in the $a b c$ frame of reference, requiring of non-existent sinusoidally distributed windings. Moreover, special knowledge of the non-conventional twoaxis approach of Adkins [5] is required to obtain the machine transfer functions. Actually, the development of conversion formulae was necessary to make a proper comparison with the complying IEEE standard results obtained in this work. It can be seen that both methods give similar results, but some discrepancies can be observed in the magnitudes of the operational reactances. These differences are indeed very remarkable because they make evident that flux-linkage harmonics are not taken into consideration in [30] due to the use of sinusoidally distributed windings. Actual distribution of stator winding is employed here, so that all harmonics produced by the machine geometry are properly considered.

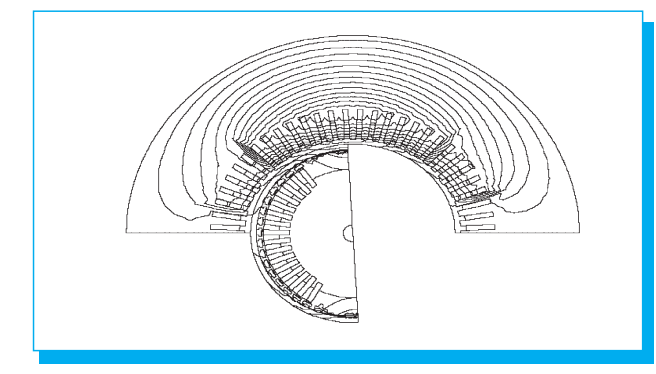

Fig. 14. Magnetic flux density distribution $\left(\omega t=0^{\circ}\right)$ at $100 \mathrm{~Hz}$. q-axis excited

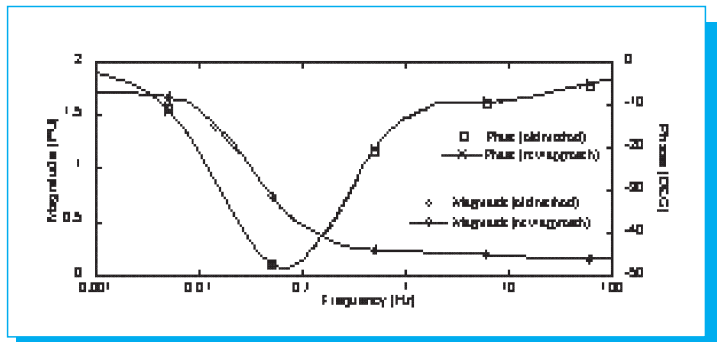

Fig. 15. $d$-axis operational reactance.

Figures 15 to 18 also show some discrepancies of the phase angles of three transfer functions at high frequencies (bigger than $60 \mathrm{~Hz}$ ). These differences are also interesting since they state that the skin depth was not correctly modelled in [30]. The reason of this assertion resides in the fact that first order elements and a coarser mesh were used in [30], making the modelling of the skin depth inaccurate. On the other hand, a very detailed/refined mesh of the rotor body (see figure 3) was constructed here at the rotor outer surface. Moreover, second order hierarchal elements were employed to improve simulation results.

\section{Conclusions}

The SSFR response of a synchronous generator was obtained with efficient and elegant finite-element techniques. Particularly, a method for the connection of the field and circuit equations was presented. This approach is based on the nodal equations of the external circuits and the total current of the finite-element conductors, such that any circuit and conductor configuration can be analysed. A very clever use of Lagrange multipliers has led to the possibility of coupling

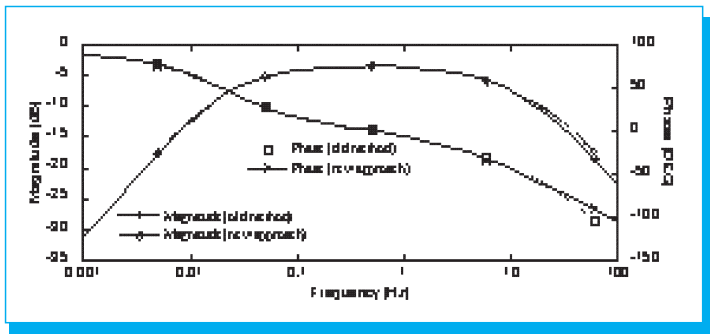

Fig. 16. $d$-axis stator current to field current transfer function. 


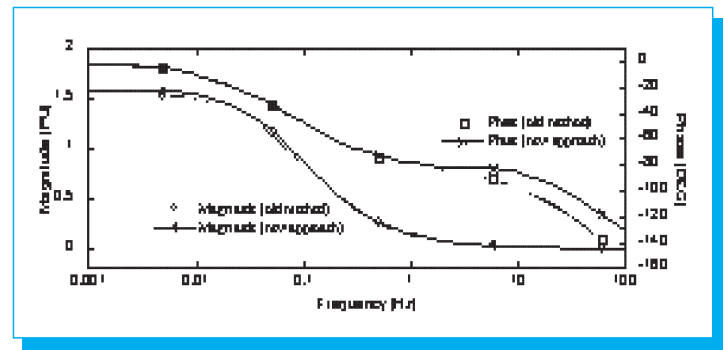

Fig. 17. Armature to field transfer reactance.

meshes of different densities, allowing nodes of elements to fall on sides of other elements. Notice that this is not permitted by the conventional finite-element theory. This way, it was possible to create a very dense mesh at the rotor outer surface, where eddy currents are induced over a small skin depth, while keeping a coarser mesh for the eddy-current free stator domain. The finite-element techniques described in this work can also be used for more general transient simulations. The determination of the SSFR test was carried out following the current IEEE standard, without resorting to non-conventional two-axis approaches or to non-existent sinusoidally distributed windings. The synchronous machine transfer functions obtained in this work were validated using proven results of other work [30], showing numerical superiority due to the use of second order elements and a better mesh.

\section{References}

[1] P.C. Krause, O. Wasynczuk and S.D. Sudhoff, Analysis of electric machinery and drive systems, IEEE Press, 2002.

[2] A.E. Fitzgerald, C. Kingsley and S.D. Umans, Electric Machinery, McGraw Hill, sixth edition, 2003.

[3] P. Kundur, Power system stability and control, Mc Graw Hill, 1994.

[4] R.H. Park, "Two-reaction theory of synchronous machines generalized method of analysis part I", AIEE Trans., Vol 48, pp. 716-727, 1929.

[5] B. Adkins and R.G. Harley, The General Theory of Alternating Current Machines, Chapman and Hall, 1975.

[6] I.M. Canay, "Modelling of alternating-current machines having multiple rotor circuits", IEEE Trans. Energy Conversion, vol. 8, no. 2, 1993, pp. 280-296.

[7] J. Verbeeck, R. Pintelon and P. Lataire, "Relationships between parameters sets of equivalent synchronous machine models," IEEE Trans. Energy conversion, vol. 14, no. 4, 1999, pp. 1075-1080.

[8] I. Kamwa, P. Viarouge, H. Le-Huy and J. Dickinson, "A frequency domain maximum likelihood estimation of

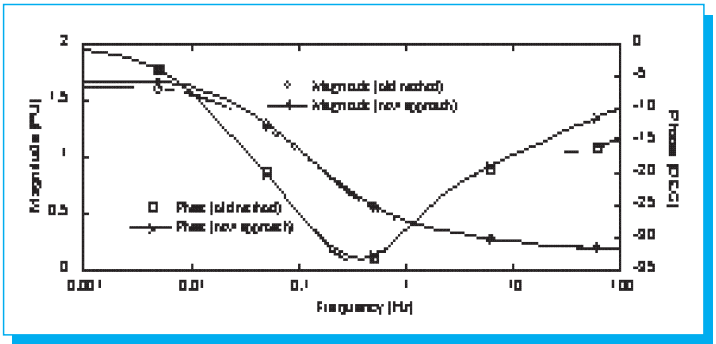

Fig. 18. $q$-axis operational reactance.

synchronous machine high-order models using SSFR test data", IEEE Trans. Energy Conversion, vol. 7, no. 3, 1992, pp. 525-536.

[9] R. Escarela-Perez and D.C. Macdonald, "A novel finiteelement transient computation of two-axis parameters of solid-rotor generators for use in power systems", IEEE Trans. Energy Conversion, vol. 13, no. 1, 1998, pp. 49-54.

[10] A. Keyhani and H. Tsai, "Identification of high order synchronous generator models from SSFR test data", IEEE Trans. Energy Conversion, vol. 9, no. 3, 1994, pp. 593-603.

[11] D.C. Aliprantis, S.D. Sudhoffand and B.T. Kuhn, "Experimental characterization procedure for a synchronous machine model with saturation and arbitrary rotor network", IEEE Trans. Energy Conversion, vol. 20, no. 3, 2005, pp. 595-603.

[12] IEEE Guide: Test Procedures for synchronous machines, IEEE Std. 115-1995.

[13] J.P.A. Bastos and N. Sadowski, Electromagnetic Modeling by finite element methods, Marcel Deker, Inc., 2003.

[14] K.J. Binns, P.J. Lawrenson, and C.W. Trowbridge, The analytical and numerical solution of electric and magnetic fields, John Wiley \& Sons, 1992.

[15] P.P. Silvester and R.L. Ferrari, Finite Elements for Electrical Engineers, Cambridge University Press, third edition, 1996.

[16] A. Konrad, "Integrodifferential finite element formulation of two-dimensional steady-state skin effect problems", IEEE Trans. Magnetics, vol. 18, no. 1, 1982, pp. 284-292.

[17] P. Lombard and G. Meunier, "A general purpose method for electric and magnetic combined problems for 2D, axisymmectric and transient systems", IEEE Trans. Magnetics, vol. 29, no. 2, 1993, pp. 1737-1740.

[18] D. Rodger, "Experience with hierarchical finite elements in 2D electromagnetics", IEEE Trans. Magnetics, vol. 23, no. 5,1987, pp. 3560-3562.

[19] S.R. Chaudhry, S. Ahmed-Zaid, and N.A. Demerdash, "Coupled finite-element/state-space modeling of 
turbogenerators in the $\mathrm{ABC}$ frame of reference - the noload case", IEEE Trans. Energy Conversion, vol. 10, no. 1, 1995, pp. 56-62.

[20] P. Zhou, W.N. Fu, D. Lin, S. Stanton and Z.J. Cendes, "Numerical modeling of magnetic devices", IEEE Trans. Magnetics, vol. 40, no. 4, 2004, pp. 1803-1809.

[21] S.J. Salon, M.J. DeBortoli, and R. Palma, "Coupling of transient fields, circuits and motion using finite element analysis", Journal of Electromagnetic Waves and Applications, vol.4, no. 11, 1990, pp. 1077-1106.

[22] P.J. Leonard, R.J. Hill-Cottingham and D. Rodger, "3D finite element models and external circuits using the A scheme with cuts", IEEE Trans. Magnetics, vol. 30, no. 5, 1994, pp. 3220-3223.

[23] T.W. Preston, A.B.J. Reece and P.S. Sangha, "Induction motor analysis by time-stepping techniques", IEEE Trans. Magnetics, vol. 24, no. 1, 1988, pp. 471-474.

[24] A.Abdel-Razek, J. Coulomb, M. Feliachi \& J. Sabonnadiere, "Conception of an air-gap element for the dynamic analysis of the electromagnetic field in electric machines", IEEE Trans. Magnetics, vol. 18, no.2, 1982, pp. 655-659.
[25] B. Davat, Z. Ren and Lajoie-Mazenc, "The movement in field modeling", IEEE Trans. on Magnetics, vol. 21, no. 6, 1985, pp. 2296-2298.

[26] D. Rodger, H.C. Lai and P.J. Leonard, "Coupled elements for problems involving movement", IEEE Trans. Magnetics, vol. 26, no. 2, 1990, pp. 548-550.

[27] E. Kreyszig, Advanced engineering mathematics, John Wiley \& Sons, Inc., seventh edition, 1993.

[28] S.H. Minnich, M.V.K. Chari and J.F. Berkery, "Operational inductances of turbine-generators by the finite-element method", IEEE Trans. Power Apparatus and Systems, vol. 102, no. 1, 1981, pp. 20-27.

[29] R. Escarela-Perez, T. Niewierowicz and E. Campero-Littlewood, "Synchronous Machine Parameters from FrequencyResponse Finite-Element Simulations and Genetic Algorithms", IEEE Trans. Energy Conversion, vol. 16, no. 2,2001,pp. 198-203.

[30] R. Escarela-Perez, E. Campero-Littlewood and T. Niewierowicz, "Efficient Finite Element Computation of Synchronous Machine Transfer Functions", IEEE Trans. Magnetics, vol. 38, no. 2, 2002, pp. 1245-1248.

\title{
Instituto Politécnico Nacional \\ Escuela Superior de Ingeniería Mecánica y Eléctrica Sección de Estudios de Posgrado e Investigación
}

\author{
9 Congreso Nacional de Ingeniería \\ Electromecánica y de Sistemas
}

Noviembre 13-172006

Sede:

Unidad Politécnica para el Desarrollo

y la Competitividad

Empresarial 